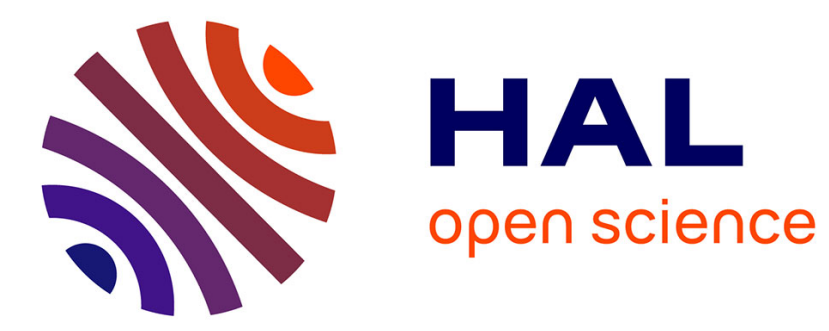

\title{
Les services biographiques répondent-ils aux motivations de récit de vie?
}

\author{
Samuel Guillemot, Bertrand Urien
}

\section{To cite this version:}

Samuel Guillemot, Bertrand Urien. Les services biographiques répondent-ils aux motivations de récit de vie?. Gérontologie et Société, 2010, 135, pp.133-151. 10.3917/gs.135.0133 . hal-00587667

\section{HAL Id: hal-00587667 \\ https://hal.univ-brest.fr/hal-00587667}

Submitted on 21 Apr 2011

HAL is a multi-disciplinary open access archive for the deposit and dissemination of scientific research documents, whether they are published or not. The documents may come from teaching and research institutions in France or abroad, or from public or private research centers.
L'archive ouverte pluridisciplinaire HAL, est destinée au dépôt et à la diffusion de documents scientifiques de niveau recherche, publiés ou non, émanant des établissements d'enseignement et de recherche français ou étrangers, des laboratoires publics ou privés. 


\title{
Les services biographiques répondent-ils aux motivations de récit de vie ?
}

\author{
Samuel Guillemot*, Doctorant en Sciences de Gestion \\ Bertrand Urien**, Professeur des Universités en Sciences de Gestion
}

Laboratoire ICI***

IAE de Bretagne Occidentale Brest

\author{
Version auteurs, \\ Article paru dans Gérontologie et Société, 2010, nº135, pages 133-151
}

Résumé:

La rédaction d'une histoire de vie par la personne âgée semble répond aux besoins fondamentaux de mémoire et de transmission. De nombreux individus se disent intéressés par la démarche d'écriture et pourtant peu passent à l'action malgré une offre de services de plus en plus développée. A partir d'une analyse lexicographique menée sur 557 comptes-rendus d'histoires de vie et de huit entretiens semi-directifs, notre recherche exploratoire permet de mieux comprendre les fonctions de ce comportement.

Mots-clés : Récit de vie, Services biographiques, Transmission intergénérationnelle, Identité

Do biographic services come up to the expectations of those wishing to write their life story?

Abstract:

For older people, writing one's life story responds to the fundamental need of memory transmission. Many people express an interest in writing but few put it into practice despite an increasing supply of services. The study explores this situation through the lexicographical analysis of 557 tales of life extracts and 8 semi-structured interviews. The results lead to a better understanding of this behavioural pattern

Key-words: Life history, Biographic services, Intergenerational transfer, Identity

\footnotetext{
*samuel.guillemot@univ-brest.fr

**bertrand.urien@univ-brest.fr

***Laboratoire ICI, IAE de Bretagne Occidentale, 12 rue de Kergoat 29238 Brest Cedex 3
} 


\section{Les services biographiques répondent-ils aux motivations de récit de vie ?}

«Votre vie offre de nombreux trésors : il serait dommage de les laisser tomber dans l'oubli... Vos proches aimeront tant s'imprégner de votre vécu pour mieux s'inscrire dans leur propre vie : faites écrire l'histoire de votre vie!» ${ }^{1}$ Il est de plus en plus fréquent de trouver ce genre de propositions commerciales dans les médias destinés aux seniors. En effet, les concepteurs de produits et services prennent peu à peu conscience des besoins et attentes résultant de l'expérience du vieillissement (Guiot, 2006, Tréguer, 2007). Par exemple, Urien et Guiot (2007) constatent le développement de produits et services permettant de gérer l'attitude face à la mort des consommateurs âgés. C'est le cas des services dit «biographiques » qui proposent aux individus les moyens de rédiger et de conserver leurs mémoires. Bien que peu ne l'écrivent, de nombreuses personnes âgées sont engagées dans l'élaboration d'une histoire de vie cohérente. Une récente enquête ${ }^{2}$ montre que près de $50 \%$ des individus ont déjà pensé à écrire leurs souvenirs mais seulement 12,5\% passent à l'action (Guillemot et Urien, 2010). Il existe donc un réel besoin que les offres de services n'arrivent pas à satisfaire. Peut-être celles-ci manquent-elles de visibilité, ? à moins qu'elles ne soient pas tout simplement pas adaptées aux attentes des personnes âgées. ? La communication autour de ces services met l'accent sur les besoins fondamentaux de mémoire et de transmission. Mais sont-ce là les seules fonctions de l'écriture de l'histoire de vie ?

L'objet de cette recherche est donc d'identifier les fonctions des histoires de vie chez les individus âgés et de les confronter aux offres de services. Dans un premier temps, nous proposons une brève approche socio-historique de la pratique des «écritures du moi » (Gusdrof, 1991) à partir d'une revue de la littérature interdisciplinaire .Puis nous dirons en quoi les personnes âgées sont particulièrement concernées. Nous présenterons ensuite une structuration des principaux services biographiques. Enfin, nous identifierons les fonctions des histoires de vie à partir de deux études empiriques :- une analyse lexicographique menée sur 557 résumés d'histoires de vie et 8 entretiens individuels semi-structurés.

\footnotetext{
${ }^{1}$ votrebiographie.com

${ }^{2}$ Etude réalisée dans les Universités du Temps Libre en Janvier 2010 auprès de 508 individus âgés de 60 à 94 ans
} 


\section{ECRITURE DU MOI ET VIEILLISSEMENT}

Les pratiques d'écriture personnelles (biographies, autobiographies, journaux, mémoires, etc.) témoignent de l'époque et la culture dans lesquelles elles se sont inscrites .. Pendant de nombreux siècles, elles n'ont pas lieu d'être car les individus distinguent difficilement leur propre conscience de la conscience collective. Avec la diffusion progressive de l'écrit et des connaissances, puis de la technologie, ces derniers prennent peu à peu la mesure de leur singularité (Pudal, 2000). C'est dans les sociétés modernes et postmodernes où les différences individuelles sont exacerbées, que s'épanouissent les écritures du moi. La pression normative pousse à être performant dans tous les aspects de sa vie (vie professionnelle, vie affective, etc.), tout en cultivant sa propre singularité, en restant «soi-même » (Delory-Momberger, 2005). Le récit permet de conjuguer ces vies multiples, en sélectionnant puis en tissant des liens entre les évènements du passée, du présent et à venir. On distingue généralement la démarche de recherche de sens qui s'incarne dans l'écriture fragmentaire (de type journaux intimes), de la construction rétrospective d'une histoire de vie cohérente (de type mémoire, autobiographie, biographie) (Pineau et Le Grand, 1993 ; Carron, 2002).

Cette dernière approche s'ancre particulièrement bien dans le processus $d u$ vieillissement. En effet, à partir de la retraite, les individus sont bien souvent sujets à un travail réflexif sur eux même et sur leur parcours de vie (Erickson, 1959). Ce retour sur soi permet d'unifier tous les aspects de sa personne, de comprendre et d'accepter sa propre vie (Butler, 1963). Ce bilan personnel en entraîne un autre, au cours duquel, la personne fait l'inventaire de ce qu'elle peut laisser sur terre, que ce soit des biens matériels ou des expériences de vie (Vercauteren et Hervy, 2002). Cela est d'autant plus vrai, qu'une crainte partagée par les personnes vieillissantes est de voir se disperser après leur mort le fruit de toute une vie (Curasi, 2006).

\section{LES SERVICES BIOGRAPHIQUES}

On comprend aisément que la mise en chantier d'une autobiographie, qui nécessite de maîtriser des compétences et un savoir-faire particuliers, puisse en décourager plus d'un. Certains auraient simplement besoin d'être aidés alors que d'autres ont besoin d'un service clé en main. D’où le développement du marché des services biographiques, en plein essor, très hétérogène, relativement peu structuré, dont il n'existe pas, à notre connaissance, de typologie. A partir de messages commerciaux et de rencontres avec des prestataires, nous 
avons recensé une quarantaine de types de services liés à l'élaboration et à la prise en charge des histoires de vie. On peut les distinguer de la façon suivante :

- Ils constituent une aide soit à l'élaboration soit à la diffusion / à la conservation du récit

- Ils sont marchands ou non marchands

- Ils sont plus ou moins interactifs (l'individu est autonome ou entre en interaction avec un tiers qui peut être virtuel)

Les services dont le but est d'aider à élaborer un récit de vie concernent les offres de formation et d'acquisition des méthodes et savoir-faire nécessaire à la rédaction d'une histoire de vie (du retour des souvenirs à la conscience, aux techniques de rédaction, en passant par la construction d'un fil conducteur). On va retrouver par exemple le cas des ateliers de réminiscence qui permettent d'aider à rappeler ses souvenirs, à les organiser et les structurer, ainsi que les ateliers d'écriture où sont dispensés des conseils de rédaction. Si ces services sont souvent non marchands et proposés par des associations, ils peuvent être également dispensés par des prestataires privés. Ces derniers, » surfant » sur la vague du coaching, proposent ainsi des offres de formation intensive (par exemple $200 €$ pour deux jours). De manière moins interactive, on peut trouver des logiciels informatiques, des manuels d'autobiographie, ou encore des livres-albums à personnaliser avec ses propres souvenirs. Dans ce cas précis, l'individu achète un bien (un livre) pour les services associés (conseils et méthodes).

D'autres prestataires proposent d'élaborer le récit de vie en collaboration : l'individu fournit alors la matière première (ses souvenirs) et le prestataire, les compétences. Il en est ainsi des écrivains professionnels. La plupart d'entre eux sont des écrivains publics, les autres sont uniquement spécialisés dans l'écriture des récits de vie. Il existerait plus de 500 biographes officiellement installés en France, et sans doute pratiquement autant de «non officiels» ${ }^{3}$. Le consommateur est confronté à toutes sortes d'offres et on y déplore malheureusement de nombreux abus. Par exemple, certains biographes privés se contenteront d'une simple retranscription des propos, tandis que d'autres effectueront un réel travail d'analyse et de structuration. Les prix varient également du simple au triple, entre 2500 et plus de $8000 €$ pour la livraison d'une biographie complète ${ }^{4}$.

\footnotetext{
${ }^{3}$ Source : Guillaume Moingeon : écrivain-biographe, www.guillaumemoingeon.com

${ }^{4}$ Magazine Lire, $\mathrm{n}^{\circ} 353$, mars 2007
} 
Les services d'édition et de diffusion prennent en charge le récit de vie lorsque celui-ci est élaboré. Des maisons d'édition proposent de publier l'auteur, le plus souvent à ses propres frais. Il existe également des offres d'auto-édition (de la mise en forme du manuscrit à son impression) où l'individu télécharge son document sur internet et personnalise des schémas préconçues (exemple : lulu.com ou les services proposés par la Fnac) Enfin, la conservation des récits est un marché émergeant. Certaines prestations de type «archives publiques »sont gratuites. On peut citer l'exemple de l'Association pour l'autobiographie (A.P.A.) où chaque texte déposé est lu « en sympathie » par un comité de lecture qui est chargé d'en faire un résumé puis archivé dans une bibliothèque .publique Le déposant peut autoriser tout un chacun à consulter son texte, ou au contraire restreindre durant une période donnée l'accès à ses archives. Des sociétés privées rendent également un service équivalent, comme le site Internet Biobble, une bibliothèque virtuelle de biographies chronologiques, agrémentées d'images, d'interviews et de dossiers thématiques, dont l'accroche est «Biobble conserve votre biographie pour toujours ».

Les tableaux 1 et 2 présentent de manière non exhaustive quelques exemples de prestations, ainsi qu'un prix indicatif.

Tableau ${ }^{\circ} 1$. Exemples de prestations de services permettant d'élaborer un récit de vie

\begin{tabular}{|c|c|c|}
\hline & Marchands & Non marchands \\
\hline Seul & $\begin{array}{l}\text { Livres-guides } \\
\text {-Conseils et méthodes, guide pas } \\
\text { à pas } \\
\text {-Achat d'un bien à forte } \\
\text { connotation de service } \\
\text { De } 10 \text { à } 20 €\end{array}$ & $\begin{array}{l}\text { Auto-formation } \\
\text { L'individu écrit seul son } \\
\text { récit. Il a la possibilité de se } \\
\text { former à travers des conseils } \\
\text { et exemples dispensés sur } \\
\text { internet }\end{array}$ \\
\hline Interactions humaines & $\begin{array}{l}\text { Stages de formations } \\
\text {-De quelques heures à plusieurs } \\
\text { jours } \\
\text { Biographes privés } \\
\text { Livraison d'un récit de vie } \\
\text { complet réalisé en collaboration } \\
\text { (écrit, audio ou vidéo) } \\
\text {-Très forte interactivité entre le } \\
\text { prestataire et le consommateur } \\
\text { De } 2000 \text { à plus de } 8000 €\end{array}$ & $\begin{array}{l}\text { Ateliers de réminiscence et } \\
\text { d'écriture } \\
\text { Animés par des praticiens en } \\
\text { établissements hébergeant } \\
\text { des personnes âgées en ou } \\
\text { par associations } \\
\text {-Souvent en groupe ; parfois } \\
\text { gratuit ou paiement à la } \\
\text { séance }\end{array}$ \\
\hline
\end{tabular}


Tableau $\mathrm{n}^{\circ} 2$. Exemples de prestations de services permettant de diffuser et de conserver un récit de vie

\begin{tabular}{|l|l|l|}
\hline Seul & Marchands & Non marchands \\
\hline & $\begin{array}{l}\text { Auto publication } \\
\text {-Téléchargement du récit sur } \\
\text { internet et personnalisation de } \\
\text { formats préconçus } \\
\text {-Prix variables selon le nombre de } \\
\text { pages et d'exemplaires }\end{array}$ & $\begin{array}{l}\text { Archives publiques } \\
\text {-Conservation du récit pour } \\
\text { les générations futures } \\
\text { Le plus souvent gratuit }\end{array}$ \\
& $\begin{array}{l}\text { Conservation sur internet } \\
\text {-Archivage de données } \\
\text { personnelles sur Internet } \\
\text {-De 50 à 200€ }\end{array}$ & $\begin{array}{l}\text { Contrat d'édition } \\
\text {-Publication et mise en vente du } \\
\text { récit de vie } \\
\text {-La plupart du temps, diffusion } \\
\text { très limitée à compte d'auteur... }\end{array}$ \\
\hline Interactions humaines & $\begin{array}{l}\text { Association pour } \\
\text { l'autobiographie (APA) } \\
\text {-Lecture « en sympathie } \\
\text { par un comité de lecture } \\
\text {-Consultation libre ou } \\
\text { restreinte selon les désirs des } \\
\text { déposants }\end{array}$ \\
\hline
\end{tabular}

Malgré une offre de services importante et un intérêt prononcé des personnes vieillissantes pour les récits de vie, peu d'entre elles passent à l'acte et concrétisent leurs souhaits. Une meilleure compréhension des motivations qui sous-tendent la rédaction d'une histoire de vie s'avère dès lors nécessaire pour développer des offres adaptées.

\section{MÉTHODOLOGIE DE LA RECHERCHE}

Afin de cerner les fonctions du récit de l'histoire de vie chez les personnes âgées, nous nous sommes appuyés sur l'Association pour l'Autobiographie (A.P.A.) ${ }^{5}$. Il s'agit d'une organisation à but non lucratif (loi 1901) qui se propose de recueillir et de conserver les histoires de vie. Elle a pour vocation de faire «vivre» les récits afin que ceux-ci rencontrent des lecteurs et constitue ainsi un «patrimoine biographique » pour les générations futures. Chaque texte déposé à l'association est lu par un comité de lecture qui est chargé d'en faire un compte-rendu, d'une dizaine de lignes à une page, publié dans un annuaire. Nous avons eu

\footnotetext{
${ }^{5}$ http://www.sitapa.org
} 
accès à 1786 de ces comptes-rendus disponibles au format numérique et avons sélectionné ceux qui faisaient référence à des textes écrits par des personnes âgées de 60 ans et plus. Au final, 557 comptes-rendus $(31,19 \%)$ répondent à ce critère avec une proportion similaire d'hommes et de femmes (respectivement 277 et 280). Les comptes-rendus collectés ont été traités à l'aide du logiciel d'analyse de données textuelles ALCESTE. Celui-ci permet de distinguer les différentes thématiques abordées dans les histoires de vie par la construction de contextes lexicaux. Un test ${\mathrm{du} \mathrm{khi}^{2}}^{2}\left(\chi^{2}\right)$ mesure la qualité d'association d'un mot avec son contexte de référence (Mathieu et Roehrich, 2005). Nous avons interprété ces thématiques à l'aide d'une revue de la littérature des auteurs en littérature « du genre autobiographique », en psychologie sociale, en sociologie du vieillissement et en gérontologie.

\section{Encadré 1 \\ Guide d'entretien}

\section{Voici quelques questions concernant votre désir biographique}

$>\quad$ Quelle histoire voudriez-vous raconter?

$>\quad$ Quelles en sont les raisons, les motivations ?

$>\quad$ Depuis quand ressentez-vous le besoin de raconter cette histoire ?

$>\quad$ Pouvez-vous lier le moment où vous avez commencé à une période ou à un événement précis?

$>\quad$ Avez-vous toujours aimé vous raconter (pour vous ou pour les autres)? Avez-vous déjà tenu un journal, des carnets (de voyage, etc.), ou tenter de rédiger une autobiographie?

$>\quad$ Comment procédez-vous ?

$>\quad$ Ressentez-vous des difficultés ?

$>\quad$ Quelles sont les destinataires de votre histoire ?

$>\quad$ Que souhaitez-vous pour votre histoire ? (impression? mise en vente ? conservation pour les générations futures? ou autres ?)

\section{La relation au passé}

$>\quad$ Vous arrive-t-il souvent de penser à votre passé ?

$>\quad$ Quels genres de souvenirs vous remémorez-vous le plus souvent ?

$>\quad$ Est-ce que vos souvenirs vous aident dans votre vie de tous les jours ? De quelle façon?

\section{La relation à la mort}

$>\quad$ Vous arrive-t-il de pensez-vous au jour où vous ne serez plus là ?, Qu'est-ce que cela évoque chez-vous ? Que faites-vous pour supporter cette idée?

\section{La relation à la jeune génération}

Quelle est votre relation avec la jeune génération?

$>\quad$ Que pensez (aimeriez)-vous pouvoir lui apporter ?

\section{Merci de votre participation !}

Voulez-vous ajouter quelque chose? 
Nous avons complété cette première étude par des entretiens semi-structurés menés auprès de personne qui ont rédigé leurs mémoires. L'enquête a été planifiée et s'est déroulée selon les recommandations de Miles et Huberman (2003).Un guide d'entretien structuré et ouvert à été élaboré à partir de la revue de la littérature (Encadré 1). Les principes de base de la non-directivité ont été respectés. La structure des entretiens n'était pas figée et l'ordre des thèmes abordés a varié selon les réponses des interviewé(e)s. Après chaque entretien une fiche de synthèse a été réalisée qui a systématiquement été comparée aux précédentes, ce qui nous a permis d'identifier le seuil de saturation. Au total, 8 personnes ont été interrogées, toutes à la retraite, âgées de 60 ans à 82 ans (moyenne de 70 ans), et couvrant le plus de situations sociales possibles (Encadré 2). Les propos ont été enregistrés et dactylographiés afin de procéder à une analyse de contenu.

\begin{tabular}{|ccccc|}
\hline \multicolumn{5}{c}{ Liste des entretiens } \\
\hline Prénom & Sexe & Age & $\begin{array}{c}\text { Profession principale } \\
\text { avant retraite }\end{array}$ & Enfants \\
\hline Sylvette & Femme & 63 ans & Bibliothécaire & Oui \\
Djamila & Femme & 63 ans & Formatrice & Non \\
Claude & Homme & 63 ans & Fonctionnaire & Non \\
Simon & Homme & 60 ans & Médecin & Oui \\
Marie & Femme & 78 ans & Enseignante & Oui \\
Marianne & Femme & 75 ans & Entrepreneuse & Oui \\
Bernard & Homme & 76 ans & Ingénieur & Oui \\
Pierre & Homme & 82 ans & Chercheur & Oui \\
\hline
\end{tabular}


PREMIÈRE ÉTUDE : LES THÈMES ABORDÉS DANS LES HISTOIRES DE VIE

L'analyse lexicale des comptes-rendus des textes déposés à l'A.P.A. fait apparaître 6 contextes sémantiques (tableau 3) commentés ci-après

Tableau 3 -Analyse lexicale des comptes-rendus des 557 textes déposés à l'A.P.A. par des personnes de 60 ans et plus

\begin{tabular}{|c|c|c|c|c|c|}
\hline \multicolumn{2}{|l|}{$\begin{array}{c}\text { Classe 1: } \\
\text { Reconnaissance }\end{array}$} & \multicolumn{2}{|l|}{$\begin{array}{l}\text { Classe2 : } \\
\text { Thérapie }\end{array}$} & \multicolumn{2}{|l|}{$\begin{array}{l}\text { Classe } 3 \text { : } \\
\text { Testament }\end{array}$} \\
\hline Vocabulaire signifiant & $\chi^{2 *}$ & Vocabulaire signifiant & $\chi^{2 *}$ & Vocabulaire signifiant & $\chi^{2 *}$ \\
\hline Etude & 262,14 & Mourir & 103,59 & Document & 167,17 \\
\hline Concours & 216,50 & Exploiter & 51,00 & Auteur & 160,20 \\
\hline Carrière & 198,59 & Malade & 41,26 & Autobiographie & 108,31 \\
\hline Obtenir & 168,04 & Quitter & 38,22 & Retracer & 90,96 \\
\hline Diplôme & 114,17 & Suicide & 35,43 & Histoire & 96,50 \\
\hline Formation & 105,27 & Dépressif & 24,28 & Parcours & 82,69 \\
\hline Devenir & 71,51 & Dur & 22,10 & Mémoire & 61,60 \\
\hline Métier & 52,98 & Veuve & 20,54 & Déposer & 38,54 \\
\hline Succès & 39,23 & Abandonner & 19,49 & Résumé & 26,32 \\
\hline Responsabilité & 33,66 & Difficile & 18,15 & Souvenir & 26,14 \\
\hline \multicolumn{2}{|l|}{$\begin{array}{c}\text { Classe 4: } \\
\text { Communauté }\end{array}$} & \multicolumn{2}{|l|}{$\begin{array}{c}\text { Classe } 5 \text { : } \\
\text { Famille }\end{array}$} & \multicolumn{2}{|l|}{$\begin{array}{c}\text { Classe 6 : } \\
\text { Patrimoine }\end{array}$} \\
\hline Vocabulaire signifiant & $\chi^{2 *}$ & Vocabulaire signifiant & $\chi^{2 *}$ & Vocabulaire signifiant & $\chi^{2 *}$ \\
\hline Amour & 163,75 & Généalogie & 210,63 & Camp & 244,37 \\
\hline Sens & 69,62 & Arbre & 205,40 & Prisonnier & 153,16 \\
\hline Exprimer & 52,98 & Génération & 129,61 & Bombardement & 151,15 \\
\hline Nature & 45,60 & Ancêtre & 116,88 & Libération & 99,41 \\
\hline Partager & 45,17 & Remonter & 106,88 & Guerre & 86,68 \\
\hline Vie & 44,52 & Grands-parents & 79,23 & Débarquement & 74,89 \\
\hline Valeur & 42,45 & Evoquer & 55,55 & Exode & 71,44 \\
\hline Foi & 37,80 & Descendant & 44,36 & Résistance & 65,99 \\
\hline Spirituel & 21,60 & Ascendant & 32,34 & Occupation & 63,25 \\
\hline Echange & 16,43 & Familial & 23,62 & Usine & 17,39 \\
\hline
\end{tabular}

\section{Reconnaissance}

L'analyse des comptes-rendus des 557 textes de l'A.P.A. révèle un premier contexte sémantique qui rassemble $13,41 \%$ du corpus et renvoie à la valorisation d'un parcours positif. Le narrateur souligne des aspects de sa vie qu'il juge dignes d'intérêt. Ce sont des réussites («succès »), des compétences («formation »), un parcours scolaire («obtenir », «diplôme ») 
ou professionnel («responsabilité », «métier», «carrière»), etc. Il ne fait nul doute que la quête de reconnaissance constitue un moteur de l'écriture personnelle. L'histoire de vie est l'occasion de faire connaître des événements, des situations vécues et supposées valorisantes (Rioul, 2003). Dans cette quete poussée à l'extrême, le narrateur peut se présenter comme « une référence ou un exemple en sélectionnant et en orientant les faits de manière à faire l'apologie de soi (Miraux, 2005).

\section{Thérapie}

Outre le fait de flatter son auteur, le récit peut servir d'exutoire à son mal-être, si bien que le second contexte $(15,29 \%)$ est marqué par des mots négatifs et porte une image douloureuse. L'histoire de vie apparaît alors comme un moyen de gérer son mal-être («suicide », «dépressif», «malade»), revendiquer sa souffrance («dur», «difficile»), et des situations douloureuses («mourir», «veuve»), etc. Il ne faut pas négliger le pouvoir libérateur du récit qui permet d'écrire ce qui a été longtemps contenu, de crever l'abcès (Houde, 1999). De manière analogue, l'histoire de vie offre la possibilité de se défendre d'accusations injustes (Hubier, 2003), de donner sa version des faits, de corriger ou démentir (May, 1979). Rousseau a été un des premiers, en son temps, à utiliser ses Confessions pour justifier sa conduite. Une illustration contemporaine nous est offerte par des octogénaires allemands qui, ayant vécu la Seconde Guerre mondiale, et se sentant défiés par la jeune génération, utilisent leur histoire de vie pour expliquer à leurs enfants que le contexte social était bien différent à l'époque (Keller, 2002).

\section{Testament}

Le troisième contexte sémantique représente $16,50 \%$ du corpus et renvoie à des «autobiographies» qui permettent de «retracer» et de «résumer» le «parcours» de l' «auteur». Comme précédemment, le narrateur est au centre du récit. Toutefois ici, la dimension posthume apparaît clairement : l'histoire de vie est un «document » à préserver («déposer », «mémoire »), les destinataires ne sont pas seulement les contemporains mais d'éventuels lecteurs dans le futur (petits ou arrières petits-enfants par exemple). Dans ce sens, le récit a pour vocation de survivre à son auteur (Caradec, 2004). Il permet au narrateur de maitriser ce qui sera retenu de lui (Collés et Duays, 1989). 


\section{Communauté}

L'histoire de vie n'a pas pour seules fonctions de parler du narrateur, de ce qu'il a fait ou de ce qu'il éprouve. Elle peut naître d'une démarche de partage. Ainsi, le quatrième contexte $(25,24 \%)$ est marqué par le relationnel («échange », «partage », « exprimer »), les valeurs («amour», «vie», «nature») et une certaine philosophie de vie («foi», «sens», spirituel »), etc. L'histoire de vie est avant tout un moyen de communication, vecteur d'échange et de partage (Janvier, 2007).

\section{Famille}

L'histoire de vie sert également à transmettre, à «passer le témoin » aux plus jeunes membres de la famille en cherchant un échange entre générations (Christen-Gueissaz, 1998). Le cinquième contexte sémantique $(8,25 \%)$ évoque les «descendants» et les «ancêtres». L'histoire de vie permet de faire connaître aux plus jeunes, les membres de la famille aujourd'hui disparus (Million-Lajoinie, 1999 ; Lecarme et Lecarme-Tabone, 1999 ; Lejeune, 2005). Dans ce sens, le récit permet aux anciens de préparer les jeunes générations à l'avenir, en leur donnant les outils et les connaissances nécessaires (Billé, 2002).

\section{Patrimoine}

Le dernier contexte sémantique représente $21,31 \%$ du corpus relatif aux comptesrendus des textes déposés à l'A.P.A. est très fortement marqué par des témoignages relatifs aux guerres et à leurs conséquences (« «occupation» «prisonnier», « résistance», «bombardement », «débarquement », «libération », etc.). Nous rappelons ici que seuls les comptes-rendus faisant références à des textes écrits par des personnes de plus de 60 ans ont été analysés. Ces derniers ont personnellement - ou par l'intermédiaire de leurs parents - vécu la seconde guerre mondiale. Le narrateur peut relater des événements dont il fut acteur ou témoin privilégié, décrire des conditions de vie symptomatiques d'une époque. L'histoire de vie est dès lors un moyen de représenter un destin collectif (Zanone, 2006) mais aussi d'y définir et d'y trouver sa place. D'une manière plus générale, elle peut servir à préserver un patrimoine (Martin Sanchez, 2003 ; Cristini et Ploton, 2009). En effet, le narrateur peut, par exemple, devenir historien de son village ou de son quartier, mettre en exergue un métier d'autrefois aujourd'hui disparu, etc. 
Les auteurs d'une biographie après 60 ans expriment des motivations qui leur sont propres et spécifiques à leur parcours de vie. Néanmoins, il est possible de les regrouper autour de deux questions centrales : pourquoi ? Et pour qui ?

L'analyse de contenu menée sur la retranscription des entretiens permet de dégager six motivations dont chacune se rattache à une fonction identifiée dans la première étude. Par exemple, un récit thérapie a pour motivation principale de «réparer l'ego » et traduit une volonté de dire une souffrance pour s'en libérer «je me dis que c'est [un moyen] de rapporter une souffrance » (Djamila), une volonté de se venger «s'ils m'avaient donné ma chance, ils n'auraient pas hérité de ce bouquin »(Claude), ou encore de rétablir une vérité «les gens de cette génération n'ont jamais connu l'histoire entière [...] je trouvais que c'était tout à fait injuste» (Marianne). De la même façon les récits «reconnaissance » peuvent s'appuyer sur une motivation à flatter l'ego «On parle de soi, c'est pour que les autres le sachent quand même » (Bernard). Les récits «famille» quant à eux relèvent principalement d'une motivation de transmission «j'ai des enfants, j'ai des petits-enfants, et j'ai envie qu'ils sachent $[\ldots]$ d'où ils viennent » (Sylvette).

Les entretiens révèlent par ailleurs une motivation de partage pour les récits « communauté » «j'ai donné un exemplaire au vétérinaire de mon chien. [...] c'est des gens que l'on côtoie pendant des dizaines d'années [...], maintenant on se connait » (Bernard),. De volonté de ne pas être oublié pour les récits «testament» «je n'ai pas d'enfant, comme ça je suis sûr que mon nom ne sera pas oublié » (Claude) .Et de témoignage pour les récits « patrimoine ». Dans ce dernier cas, nos entretiens révèlent des mobiles aussi variés qu'un témoignage sur l'évolution du $\mathrm{XX}^{\text {ème }}$ siècle (Bernard), sur un fait de société comme le racisme (Djamila), sur un engagement idéologique (Claude), sur l'évolution d'un métier (Simon), ou d'une manière plus générale une volonté de contribuer modestement à la connaissance universelle "il y a des tas de sources qui intéressent les sociologues et historiens. C'est un aspect intéressant de la chose, on sait qu'on est un matériau contributif, en quelque sorte » (Pierre).

Outre les différentes attentes inhérentes à l'élaboration d'une histoire de vie, l'existence de lecteurs semble toujours souhaitée. Ces derniers peuvent-être regroupés en quatre familles: des personnes clairement identifiées, le plus souvent les enfants et les petits- 
enfants de la famille, plus généralement les proches ou encore «des collègues [...] et des connaissances, sans plus »(Bernard) ; des personnes identifiées non nommées, par exemple les anciens clients de l'entreprise familiale (Marianne), les membres d'une association (Sylvette) ou encore les habitants d'une commune (Claude) ; des lecteurs non identifiés (des inconnus), surtout lorsqu'on a le désir de se faire publier «C'est pour les gens qui vivent ici, en Europe et qui se font une idée un peu angélique du reste du monde » (Djamila); , des lecteurs potentiels au-delà de la mort «C'est un peu comme ça, je laisse des choses, parce qu'actuellement il n'y a pas d'oreilles pour m'entendre »(Claude), ou encore des chercheurs «C'est un peu ça aussi, laisser des matériaux pour les historiens »(Claude).

Le tableau 4 met en perspective nos résultats. Il convient néanmoins de préciser qu'une même thématique peut-être abordée pour plusieurs motivations distinctes. Et que les mêmes motivations peuvent aboutir à des thématiques différentes. Toutefois, les résultats issus de deux études très différentes (des entretiens « en profondeur » et un corpus très large de 557 comptes-rendus de récit de vie) convergent et tendent à valider une structure à six dimensions.

Tableau $\mathrm{n}^{\circ} 4$ - Une typologie des histoires de vie et de leurs fonctions

\begin{tabular}{|l|l|l|}
\hline $\begin{array}{c}\text { Type de récit } \\
\text { (étude 1) }\end{array}$ & \multicolumn{1}{|c|}{$\begin{array}{c}\text { Motivations } \\
\text { (étude 2) }\end{array}$} & \multicolumn{1}{c|}{$\begin{array}{c}\text { Destinataires } \\
\text { (étude 2) }\end{array}$} \\
\hline Reconnaissance & Flatter l'ego & $\begin{array}{l}\text { Des proches } \\
\text { Des inconnus }\end{array}$ \\
\hline Thérapie & Réparer l'ego & $\begin{array}{l}\text { Des proches } \\
\text { Des inconnus } \\
\text { Des personnes « ciblées » }\end{array}$ \\
\hline Testament & $\begin{array}{l}\text { Ne pas être } \\
\text { oublié }\end{array}$ & $\begin{array}{l}\text { Des lecteurs au-delà de la mort } \\
\text { inconnus ou proches }\end{array}$ \\
\hline Communauté & Partager & $\begin{array}{l}\text { Des proches } \\
\text { Des personnes identifiées } \\
\text { Des inconnus }\end{array}$ \\
\hline Famille & Transmettre & $\begin{array}{l}\text { Des proches } \\
\text { Des personnes identifiées }\end{array}$ \\
\hline Patrimoine & Témoigner & $\begin{array}{l}\text { Des lecteurs au-delà de la mort } \\
\text { Des chercheurs }\end{array}$ \\
\hline
\end{tabular}

L'adéquation entre les attentes du narrateur et l'intérêt des destinataires est un élément important qui concourt à la réalisation du récit. Un soutien moral entraîne plus d'efforts mobilisés et plus de persévérance; «pour mes petits-enfants, j’ai ressorti des notes de Noël des années 60, [...] ça les a intéressés, ils voulaient savoir la suite, ils veulent d'autres 
histoires comme ça»(Pierre). Au contraire, Djamila éprouve des difficultés à terminer son histoire car elle ne sait pas à qui 1'adresser «Bon je travaille, mais je travaille moins qu'avant. Le problème c'est d'arriver à intéresser quelqu'un [...] au fond de moi, c'est 'a quoi bon'».

\section{DISCUSSION}

L'objet de cette recherche était de confronter les offres de services aux fonctions des histoires de vie chez les personnes âgées. A partir d'une revue de la documentation commerciale, nous avons proposé une structure au marché des services biographiques. Puis deux études qualitatives, une analyse lexicographique menée sur 557 comptes-rendus d'histoire de vie et 8 entretiens semi-directifs, nous ont permis de préciser les fonctions des histoires de vie. Certaines sont très liées à la notion d'identité (diffuser et communiquer une image valorisée de soi, réparer l'ego) et à la préservation de celle-ci (ne pas être oublié), d'autres sont d'avantage liées aux relations intragénérationnelles (partager) et intergénérationnelles (témoigner, transmettre).

Ainsi, si certaines fonctions des histoires de vie sont bien prises en compte par les prestataires, d'autres sont négligées. Les personnes âgées ne valorisent pas seulement l'écriture de vie pour préserver et transmettre une part de leur expérience de vie pour les générations futures mais utilisent également, comme chez les plus jeunes, la production de soi comme technique relationnelle. Ainsi, les prestataires de services doivent valoriser cette fonction et mettre en avant l'interactivité et l'échange dans leur offre. Dans ce sens, une des clés du succès de l'A.P.A est que le service offert porte sur deux dimensions importantes du récit de vie : le narrateur à la garantie, s'il n'interdit pas la consultation de son texte, d'être lu, à minima, par le comité de lecture, ainsi que de voir inscrire son œuvre dans le Gardemémoire de l'association. Le discours des personnes interrogées lors des entretiens qualitatifs ${ }^{6}$ montre que le dépôt à l'A.P.A. est bien souvent envisagé comme une alternative à une diffusion plus large (entendue ici au sens d'édition) comme l'illustre les verbatim suivants : "Ce texte même s'il n'est édité il sera au moins dans les archives »(Pierre), «Dans mon livre je parle des méfaits du Communisme, c'est un sujet qui fâche! [...] Actuellement il n'y a pas d'oreille pour m'entendre, j'ai l'espoir que mon texte intéressera les chercheurs [...] dans 50 ans je ne serai plus là mais mon texte il sera toujours à l'A.P.A. ! »

\footnotetext{
${ }^{6} 6$ des 8 personnes interrogées ont déposés leur manuscrit dans les archives de l'APA
} 
L'interactivité est également à chercher dans les activités de groupe comme, par exemple, les ateliers d'écriture. Les individus y viennent, certes pour améliorer leur style, mais également pour faire «vivre » les textes. L'échange, le fait d'être écouté, lu, stimulé et encouragé, mais aussi la possibilité de réagir aux histoires des autres constituent des éléments déterminants dans la fréquentation des ateliers de groupe. D'ailleurs les ateliers de partage des souvenirs proposés dans certains établissements hébergeant des personnes âgées sont souvent des succès. Ils permettent de créer un lien social entre les résidents (Billé, 2006 ; Ribes et alii, 2009), entre les résidents et le personnel (Ross, 1990 ; Trueman et Parker, 2006) et parfois même entre les résidents et leurs propres familles (Argentin, 2006).

Garantir la qualité de vie des personnes dépendantes est un enjeu de société pour le $\mathrm{XXI}^{\text {ème }}$ siècle. Il convient de placer les personnes vivant en établissement au centre des préoccupations de tous les professionnels, en les considérant, non seulement comme des sujets mais également comme des consommateurs. Il s'agira alors de répondre à leurs besoins et attentes (Leclet et Vilcot, 2008). En ce sens, l'animation est un axe stratégique et devient un véritable argument commercial pour les établissements privés (Hervy, 2003). L'animation auprès des personnes âgées consiste à «faciliter l'insertion des anciens dans la société, leur participation à la vie sociale, et surtout leur permettre d'exercer un rôle, voire d'engager un processus de réactivation des rôles sociaux, seule réponse à une mort sociale qui intervient parfois avant la mort biologique » (Hartweg et Zehnder, 2003). Ainsi, encourager la personne à s'exprimer et à construire un récit de vie est une activité qui a toute sa place en établissement hébergeant des personnes âgées dépendantes (Vercauteren et Hervy, 2002 ; Sudres et alii, 2004). Il serait pertinent, par exemple, de proposer des ateliers ludiques autour des souvenirs qui utilisent les leviers de partage et de transmission. L'objectif est de proposer par la suite, et pour ceux qui le souhaitent, des services d'apprentissage plus poussés ou des services de co-production.

Il convient bien évidemment de considérer l'éthique de telles pratiques. Nous avons ainsi vu qu'une même prestation pouvait être facturée du simple au triple. Une meilleure connaissance et une plus grande visibilité des services biographiques permettront sans doute d'enrayer ce phénomène. Outre l'aspect financier, il existe un risque moral et psychologique à se raconter devant un ou des inconnu(s). Le prestataire doit être formé pour gérer des situations potentiellement bouleversantes (un individu qui évoque des souvenirs douloureux) et doit pouvoir fixer des limites claires (il n'est pas un psychothérapeute). Ainsi, ce métier nécessite des compétences littéraires, des qualités d'écoute, de compréhension et d'empathie. Au delà, la diffusion de récit s'inscrit dans un cadre légal: "A-t-on le droit de tout dire ? ». Les 
récits de vie reposent en effet sur l'authenticité des souvenirs rapportés de la «vraie vie » et s'accompagnent bien souvent de détails géographiques et de précisions sur les acteurs impliqués. Au-delà des nombreux débats autour de la vérité narrative (Pillemer, 1998)' on ne peut que constater la multiplication des assignations en diffamation par des personnes qui croient se reconnaitre ou qui ne veulent pas qu'un aspect de leur vie soit divulgué sur la place publique. Les prestataires de services biographiques doivent se positionner clairement et un contrat réglant certaines de ces questions est nécessaire (Pineau et Legrand, 1993). Par exemple, il est indispensable pour un biographe privé de connaitre les destinataires du récit (sphère privée ou sphère publique ?) et celui qui sera considéré comme l'auteur (le prestataire qui écrit ou l'individu qui raconte?).

La présente recherche comporte quelques limites. Premièrement, nous avons mené l'analyse lexicographique sur des comptes-rendus d'histoires de vie réalisés par un comité de lecture et non sur le récit complet. des auteurs.Toutefois ces comités de lecture sont composés de membres de l'APA et ont une connaissance très fine de ce type de récit. Ils sont par ailleurs tenus au respect d' une charte qui garantit une cohérence dans le traitement des dépôts et dans le contact avec les déposants. Cette limite est atténuée par les résultats de la seconde étude qui confirment, précisent et illustrent les fonctions d'une histoire de vie. Par ailleurs, nous n'avons pas examiné les effets de cohorte. Les événements socio-historiques survenus pendant les années où s'opèrent les choix de vie (15-25 ans) forment les attitudes et les comportements d'une génération (Préel, 2000). Ainsi la cohorte 1920-1930 qui a vécu et participé à la guerre a sans doute des faits et des histoires différentes à raconter par rapport à la cohorte 1945-1955 qui a grandi avec la croissance économique (Lalive d'epinay et Cavalli, 2009). Nous pensons néanmoins que la différence se ferra d'avantage dans le contenu de l'histoire que sur le plan de la nature des motivations. Ainsi, la fonction témoigner est commune à toutes les cohortes, les plus âgés témoignant sur la guerre, les plus jeunes sur des idéologies ou d'autres évènements (Mai 68, etc.). Une autre voie de recherche nécessaire et utile d'un point de vue managérial est d'identifier les représentations qu'ont les consommateurs des services biographiques. Les représentations sont constituées des connaissances, objectives ou subjectives que possède le consommateur sur le service considéré (Mathieu, 2002). En effet, si la plupart des individus a une idée générale des services biographiques, la majorité ne saurait encore décrire le déroulement et le contenu d'une prestation. 
BIBLIOGRAPHIE

Argentin L. (2006), Parler de son temps: récits de vie et ateliers d'écritures, Soins Gérontologie, 57, 38-42

Billé M. (2002), A quoi servent les grands-parents? Des grands-parents pour introduire au « sacré », Dialogue, 158, 3-10

Billé M. (2006), Le lien social au cœur de la prévention, Le traitement social de la vieillesse, La gérontologie en actes, L'Harmattan, 71-84

Butler R.N. (1963), The Life Review: an interpretation of reminiscence in the age, Psychiatry, 26, 65-75

Cappeliez P. (2009), Fonctions des réminiscences et dépression, Gérontologie et Société, $130,171-186$

Caradec V. (2004), Vieillir après la retraite, Approche sociologique du vieillissement, PUF

Carron J-P. (2002), Ecriture et identité, pour une poétique de l'autobiographie, Edition Ousia

Christen-Gueissaz E. (1998), Mémoire et récit de vie chez les adultes âgés, Gérontologie, $105,1,31-39$

Collés L. et Duays J-L. (1989), Le récit de vie, Vade-mecum du professeur de français, Bruxelles, Didier Halder

Cristini C. et Ploton L. (2009), Mémoire et autobiographie, Gérontologie et Société, 130, $75-95$

Curasi C.F. (2006), Maybe it is your father's Oldsmobile: the construction and preservation of family identity through the transfer of possessions, Advances in Consumer Research, 33, $82-86$

Delory-Momberger C. (2005), Histoire de vie et Recherche biographique en éducation, Economica

Erikson E.H. (1959), Identity and the life cycle: Selected papers, Psychological Issues, 1, 50100

Guiot D. (2006), Un cadre d'analyse du processus de vieillissement et de son influence sur le comportement d'achat du consommateur âgé, Recherche et Applications en Marketing, 27, $1,57-79$

Guillemot S. et Urien B. (2010), La rédaction d'une histoire de vie chez les personnes âgées : fondements conceptuels, dimensionnement et proposition d'une échelle de mesure des motivations au récit de vie, Recherche et Applications en Marketing, 25, 4, 25-43

Gusdorf G. (1991), Lignes de vie - Les écritures du moi (Tome 1), Editions Odile Jacob 
Hartweg C. et Zehnder G. (2003), Animateurs et animation en établissements pour personnes âgées, Pratiques gérontologiques, Eres édition

Hervy B. (2003), Propositions pour le développement de la vie sociale des personnes âgées, rapport de la mission «vie sociale des personnes âgées », Edition de 1'Ecole National de la Santé Publique

Houde R. (1999), Les temps de la vie, Le développement psychosocial de l'adulte, Morin, $3^{\text {ème }}$ édition

Hubier S. (2003), Littératures intimes : les expressions du moi, de l'autobiographie à l'autofiction, A. Colin

Janvier M. (2007), Atelier d'écriture et personnes âgées, Aux vendanges de la vie, Chronique Sociale, Lyon

Keller B. (2002), Personal Identity and Social Discontinuity of the "War Generation" in Former West Germany, In Webster et Haight (Eds), Critical Advances in Reminiscence Work : From Theory to Application, Springer Publishing Company, 165-179

Lalive d'Epinay C. et Cavalli S. (2009), Mémoire de l'histoire et appartenance générationnelle des personnes âgées, Gérontologie et société, 130, 127-144

Lecarme J. et Lecarme-Tabone E. (1999), L'autobiographie, $2^{\text {nd }}$ édition, Armand Colin, Paris

Leclet H. et Vilcot C. (2008), Qualité en gérontologie, Afnor éditions, $2^{\text {nd }}$ édition

Lejeune P. (2005), Signes de vie : Le pacte autobiographique 2, Edition du Seuil, Paris

Martin Sanchez M-O (2003), Discours oraux, discours écrits, les séniors et l'écriture de soi, Gérontologie et société, 106, 97-109

Mathieu J.P. et Roehrich G. (2005), Les trois représentations du marketing au travers de ses définitions, Revue Française du Marketing, 204, 39-56

May G. (1979), L'autobiographie, PUF, Paris

Miles A.M. et Huberman M.B. (2003), Analyse des données qualitatives, De Boeck

Million-Lajoinie M.M (1999), Reconstruire son identité par le récit de vie, L'Harmattan, Paris

Miraux J-P. (2005), L'autobiographie, écriture de soi et sincérité, Armand Colin

Pillemer D. (1998), Momentous events, vivid memories : How unforgettable moments help us understand the meaning of our lives, Cambridge, MA : Harvard University Press

Pineau G. et Le Grand J-L. (1993), Les histoires de vie, PUF, collection « Que sais-je », $2^{\text {nd }}$ édition 2002

Préel B. (2000), Le choc des générations, Editions la Découverte, Paris 
Pudal B. (2000), Ecritures non professionnelles et prises de parole, Les Actes de Lecture, 69, 93-96

Ribes G., Sagne A., Gaucher J. et Ploton L. (2009), Mémoire générationnelle et identité, Gérontologie et Société, 130, 145-153

Rioul R. (2003), Le désir autobiographique, Conférence prononcée le 7 mars dans le cadre de l’Université du Temps Libre, Université Marc-Bloch, Strasbourg

Ross H.K (1990), Lesson of life, Geriatric Nursing, 11, 274-275

Sudres J-L., Roux G., Laharie M., et de la Fournière F. (2004), La personne âgée en ArtThérapie, de l'expression au lien social, Paris, L'Harmattan

Tréguer J-P. (2007), Le senior Marketing, Dunod, Paris, 4ème édition

Trueman I. et Parker J. (2006), Exploring community nurses' perceptions of life review in palliative care, Journal of Clinical Nursing, 15, 197-207

Urien B. et Guiot D (2007), Attitude face à la mort et comportement d'ajustement des consommateurs âgés : Vers l'élaboration d'une réponse marketing, Décision Marketing, 46, 23-35

Vercauteren R. et Hervy B. (2002), L'animation dans les établissements pour personnes âgées, Manuel des pratiques professionnelles, Pratiques gérontologiques, Eres édition

Zanone D. (2006), L'autobiographie, Ellipses 